\title{
NUCLEARITY OF OPERATORS RELATED TO FINITE MEASURE SPACES
}

\author{
VISHVESH KUMAR AND SHYAM SWARUP MONDAL
}

\begin{abstract}
Let $(S, \mathcal{B}, m)$ be a finite measure space. The aim of this paper is to give necessary and sufficient conditions on symbols such that the corresponding $\mathbb{Z}$-operator from $L^{p_{1}}(\mathbb{Z})$ into $L^{p_{2}}(\mathbb{Z})$ and $S$-operator from $L^{p_{1}}(S)$ into $L^{p_{2}}(S)$ to be nuclear for $1 \leq p_{1}, p_{2}<\infty$. We show that the adjoint of the nuclear $\mathbb{Z}$-operator from $L^{p_{2}^{\prime}}$ into $L^{p_{1}^{\prime}}$ is again a nuclear operator. As applications, we get the symbol of the product of the nuclear operators with bounded operators.
\end{abstract}

\section{INTRODUCTION}

The theory of pseudo-differential operators is one of the most important tools in modern mathematics. Pseudo-differential operators are widely used in harmonic analysis, PDEs, geometry, mathematical physics, time-frequency analysis, imaging and computations [23. The study of pseudodifferential operators is first introduced by Kahn and Nirenberg [25] and later used by Hörmander 23. for problems in partial differential equations. Pseudo-differential operators on different classes of groups are broadly studied by several authors with their applications [5, 6, 7, 8, 11, 12, 20, 26, 27, 28, 29, 37, 39, 40, 41, 42, 43.

In particular, the pseudo-differential operators on the lattice $\mathbb{Z}^{n}$ are suitable for solving difference equations on $\mathbb{Z}^{n}$. Discrete pseudo-differential operators appear early in the classic Fourier analysis as well as discrete counterparts of Calderoń-Zygmund singular integral operators. A suitable theory of pseudo-differential operators on the lattice $\mathbb{Z}^{n}$ has been developed by several authors but without symbolic calculus [3, 18, 31, 34, 35. Very recently, a global symbolic calculus has been introduced and constructed by Botchway, Kabiti and Ruzhansky with many applications [3.

The idea of formulation a global definition of pseudo-differential operators on the unit circle $\mathbb{S}^{1}$ using Fourier series was first proposed by Agranovich crediting Volevich [1. Later, this theory was developed by Turunen and Vainikko [42, and Ruzhansky and Turunen 39. Though, some interdependent results for this periodic theory is given in the works of Ruzhansky and Turunen [39, 40, Cardona [4, 8, Delgado [14] and Molahajloo and Wong [30, 32].

For the trace class operators on Hilbert spaces, in general the trace which is given by the integration of its kernel over the diagonal is equal to the sum of its eigenvalues. However, this feature fails in Banach spaces. The importance of nuclear operator lies in the work of Grothendieck, who proved that for 2/3-nuclear operators, the trace in Banach spaces agrees with the sum of all the eigenvalues with multiplicities counted. Therefore, the notion of nuclear operators becomes useful. Nuclear operators on Banach spaces as generalizations of trace class operators can be traced at least to Grothendieck [21, 22, The initiative of finding the necessary and sufficient conditions for a pseudo-differential operator defined on a group to be nuclear has been started by Delgado and Wong 18 . The nuclearity of pseudo-differential operators on $\mathbb{R}^{n}$ has been studied by Aoki 2 and Rempala [36]. Characterizations of nuclear operators in terms of decomposition of symbol through

Date: June 27, 2021.

2010 Mathematics Subject Classification. Primary 47F05, 47G30; Secondary 43A85,

Key words and phrases. Pseudo-differential operators; Finite measure space; Fourier transform; $S$-operators; $\mathbb{Z}$ operators. 
Fourier transform were investigated by Ghaemi, Jamalpour Birgani and Wong for $\mathbb{S}^{1}$ 19. Later they generalized their results on nuclearity to the pseudo-differential operators for any arbitrary compact group [20. On the other hand characterizations of nuclear operators in terms of decomposition of symbol for $\mathbb{Z}$ was studied by Jamalpour Birgani [24]. Moreover $L^{p}$-boundedness and $L^{p}$-nuclearity of multilinear pseudo-differential operators on $\mathbb{Z}^{n}$ and the tours $\mathbb{T}^{n}$ proved by D. Cardona and the first author in [7]. However, a more general result, $L^{p}$-nuclearity and its traces of pseudo-differential operators on compact Lie groups, compact homogeneous spaces and compact manifolds studied by Delgado and Ruzhansky [13, 15, 16, 40].

The motivation to study $S$-operator and $\mathbb{Z}$-operators comes from the Fourier analysis associated with the classical orthogonal polynomials expansions on bounded interval or on half real line. The pseudo-differential operator on the circle $\mathbb{S}^{1}$ or on the lattice $\mathbb{Z}$ defined using the usual Fourier analysis on $\mathbb{S}^{1}$ or on $\mathbb{Z}$. In the same way we can define pseudo-differential operator with the help of the Fourier analysis associated with the classical orthogonal polynomials expansion on bounded interval or on half real line. Here, we work in a more general setting by considering a finite measure spaces $S$ equipped with a measure $m$ such that $m(S)<\infty$ such that $L^{2}(S)$ is a separable Hilbert space and define the pseudo-differential operators with the help of the orthonormal basis of separable Hilbert space $L^{2}(S)$. Such operators were first introduced by Catană as $S$-operators and $\mathbb{Z}$-operators related to the measure space $S$ which are generalization of pseudo-differential operators on $\mathbb{S}^{1}[30$ ] and $\mathbb{Z}\left[31\right.$ respectively [9, 10]. Properties such as $L^{2}$-boundedness, compactness of $S$-operator and $\mathbb{Z}$-operator are given in [9, 10].

In this paper, we consider that $\left\{e_{n}\right\}_{n \in \mathcal{I}}$ with $\mathcal{I}=\mathbb{Z}$ or $\mathbb{N}$ is an orthonormal basis for the separable Hilbert space $L^{2}(S)=L^{2}(S, \mathcal{B}, m)$ with an extra property that for every $n \in \mathcal{I}, m\left\{s \in S: e_{n}(s)=\right.$ $0\}=0$. Although, by following the notation in [9, [10] we prefer to write $\mathcal{I}=\mathbb{Z}$ in this paper but our results also true when $\mathcal{I}=\mathbb{N}$ which is the case of many interesting examples discussed below other than circle $\mathbb{S}^{1}$. Examples of such kind of finite measure spaces for which their $L^{2}$-Hilbert spaces are separable and the corresponding orthonormal basis owns this property are as follows: let us consider the triple $(S, \mathcal{B}, m)$, where $S$ is the interval $(a, b), \mathcal{B}$ is the set of all Baire subsets in $(a, b)$ and $m$ is a Baire measure.

(1) When $a=-1, b=1$ and $m(d s)=d s$ is the standard Lebesgue measure, consider the orthogonal sequence of Legendre polynomials

$$
\left\{P_{n}(x)=\frac{1}{2^{n} n !} \frac{d^{n}}{d x^{n}}\left(x^{2}-1\right)^{n}\right\}_{n \geq 0} \text { on }(-1,1) .
$$

Then, the set

$$
\left\{e_{n}=\frac{P_{n}}{\left\|P_{n}\right\|}=\sqrt{\frac{2 n+1}{2}} P_{n}\right\}_{n \geq 0}
$$

is an orthonormal basis for $L^{2}(-1,1)$ with the standard inner product $(\cdot, \cdot)$ and the norm $\|\cdot\|$.

(2) When $a=-\infty, b=\infty$ and $m(d s)=e^{-s^{2}} d s, s \in S=\mathbb{R}$, consider the orthogonal sequence of Hermite polynomials

$$
\left\{H_{n}(x)=(-1)^{n} e^{x^{2}} \frac{d^{n}}{d x^{n}}\left(e^{-x^{2}}\right)\right\}_{n \geq 0} \text { on } \mathbb{R} \text {. }
$$

So,

$$
\left\{e_{n}=\frac{H_{n}}{\left\|H_{n}\right\|}=\sqrt{\frac{n !}{2^{n} \sqrt{\pi}}} H_{n}\right\}_{n \geq 0}
$$


is an orthonormal basis for $L^{2}(\mathbb{R})$.

(3) When $a=0, b=\infty$ and $m(d s)=e^{-s} d s$, consider the sequence of orthogonal Laguerre polynomials

$$
\left\{L_{n}(x)=\frac{e^{x}}{n !} \frac{d^{n}}{d x^{n}}\left(x^{n} e^{-x}\right)\right\}_{n \geq 0} \quad \text { on }(0, \infty)
$$

then the set

$$
\left\{e_{n}=\frac{L_{n}}{\left\|L_{n}\right\|}=L_{n}\right\}_{n \geq 0}
$$

is an orthonormal basis for $L^{2}(0, \infty)$.

The main aim of this paper is to study the nuclear $\mathbb{Z}$-operators and $S$-operators related to a finite measure space with application to adjoints and products. We give necessary and sufficient conditions on the symbols such that the corresponding $\mathbb{Z}$-operators and $S$-operator to be a nuclear operator. Further we provide necessary and sufficient conditions on the symbols to guarantee that the adjoint and the product of nuclear $\mathbb{Z}$-operators are nuclear.

The schema of the paper is as follows: In section 2, we recall $S$-operators and $\mathbb{Z}$-operators (also known as the generalized pseudo-differential operator on $S$ and $\mathbb{Z}$ ) related to a finite measure space $(S, \mathcal{B}, m)$ from [9, 10. We begin section 3 by the definition of nuclear operators on Banach space and present a characterization of nuclear $\mathbb{Z}$-operators. We also give necessary and sufficient conditions on the symbols to guarantee that the adjoints and products of nuclear $\mathbb{Z}$-operators are nuclear. Characterization of nuclear $S$-operator are given in Section 4. We end this paper by presenting a few examples of $S$-operator and $\mathbb{Z}$-operator corresponding to some finite measure spaces for which the results in Section 3 and Section 4 are valid.

\section{Preliminary}

In this section, we recall some basic facts related to the Fourier analysis on finite measure space $(S, \mathcal{B}, m)$ developed by Catană in $[9,10$. Let $(S, \mathcal{B}, m)$ be a finite measure space (i.e. the total measure of $S, m(S)$ is finite) such that the corresponding Hilbert space $L^{2}(S)=L^{2}(S, \mathcal{B}, m)$ is separable. For $f, g \in L^{2}(S)$, the inner product on Hilbert space $L^{2}(S)$ is given by

$$
(f, g)_{L^{2}(S)}=\int_{S} f(s) \overline{g(s)} d m(s) .
$$

Let $\left\{e_{n}\right\}_{n \in \mathbb{Z}}$ be an orthonormal basis for $L^{2}(S)$. On the other hand $L^{2}(\mathbb{Z})$ is a Hilbert space with respect to the inner product is given by

$$
(a, b)_{L^{2}(\mathbb{Z})}=\sum_{n=-\infty}^{\infty} a_{n} \overline{b_{n}}, \quad a, b \in L^{2}(\mathbb{Z}) .
$$

We define the linear operators $\mathcal{F}_{S}: L^{2}(S) \rightarrow L^{2}(\mathbb{Z})$ and $\mathcal{F}_{\mathbb{Z}}: L^{2}(\mathbb{Z}) \rightarrow L^{2}(S)$, by

$$
\left(\mathcal{F}_{S} f\right)(n)=(m(S))^{-1 / 2}\left(f, e_{n}\right)_{L^{2}(S)}, \quad n \in \mathbb{Z},
$$

for all $f$ in $L^{2}(S)$ and

$$
\left(\mathcal{F}_{\mathbb{Z}} a\right)(s)=(m(S))^{1 / 2} \sum_{n \in \mathbb{Z}} a_{n} e_{n}(s), \quad s \in S,
$$

for all sequence $a$ in $L^{2}(\mathbb{Z})$. The linear operators $\mathcal{F}_{S}$ and $\mathcal{F}_{\mathbb{Z}}$ are bijections and they are the inverses to each other. So,

$$
\mathcal{F}_{S} \mathcal{F}_{Z}=I: L^{2}(\mathbb{Z}) \rightarrow L^{2}(\mathbb{Z})
$$


and

$$
\mathcal{F}_{\mathbb{Z}} \mathcal{F}_{S}=I: L^{2}(S) \rightarrow L^{2}(S),
$$

where we denoted by the same capital letter $I$, the identity operator of $L^{2}(\mathbb{Z})$ and $L^{2}(S)$ respectively. Moreover, these operators also satisfy the following version of the Plancherel formula.

Theorem 2.1. For all $f$ and $g$ in $L^{2}(S)$, we have

$$
\left(\mathcal{F}_{S} f, \mathcal{F}_{S} g\right)_{L^{2}(\mathbb{Z})}=(m(S))^{-1}(f, g)_{L^{2}(S)}
$$

and for all $a$ and $b$ in $L^{2}(\mathbb{Z})$, we have

$$
\left(\mathcal{F}_{\mathbb{Z}} a, \mathcal{F}_{\mathbb{Z}} b\right)_{L^{2}(S)}=m(S)(a, b)_{L^{2}(\mathbb{Z})} .
$$

Let $\sigma$ be a measurable function on $S \times \mathbb{Z}$. Then for all $f$ in $L^{2}(S)$, we define the function $T_{\sigma} f$ formally by

$$
\left(T_{\sigma} f\right)(s)=(m(S))^{1 / 2} \sum_{n \in \mathbb{Z}} \sigma(s, n)\left(\mathcal{F}_{S} f\right)(n) e_{n}(s), \quad s \in S,
$$

where the linear operator $\mathcal{F}_{S} f$ is given in 2.1 . We call $T_{\sigma}$ the $S$-operator or the generalized pseudodifferential operator on $S$ [related to the measure space $(S, \mathcal{B}, m)$ ] corresponding to the symbol $\sigma$, whenever the series is convergent for all $s \in S$.

Again, let $\tau: \mathbb{Z} \times S \rightarrow \mathbb{C}$ be a measurable function. Then for every sequence $a$ in $L^{2}(\mathbb{Z})$, we define the sequence $T_{\tau} a$ formally by

$$
\left(T_{\tau} a\right)(n)=(m(S))^{-1 / 2} \int_{S} \tau(n, s)\left(\mathcal{F}_{\mathbb{Z}} a\right)(s) \overline{e_{n}(s)} d m(s), n \in \mathbb{Z},
$$

where the linear operator $\mathcal{F}_{\mathbb{Z}} a$ is given in 2.2 . We call $T_{\tau}$ the $\mathbb{Z}$-operator or the generalized pseudodifferential operator on $\mathbb{Z}$ [related to the measure space $(S, \mathcal{B}, m)]$ corresponding to the symbol $\tau$, whenever the integral exists for all $n \in \mathbb{Z}$. See [9, 10] for more details about $S$ and $\mathbb{Z}$-operators.

\section{3. $\mathbb{Z}$-OPERATORS}

We first recall the basic notions of nuclear operators on Banach spaces. Let $T$ be a bounded linear operator from a complex Banach space $X$ into another complex Banach space $Y$ such that there exist sequences $\left\{x_{n}^{\prime}\right\}_{n=1}^{\infty}$ in the dual space $X^{\prime}$ of $X$ and $\left\{y_{n}\right\}_{n=1}^{\infty}$ in $Y$ such that

$$
\sum_{n=1}^{\infty}\left\|x_{n}^{\prime}\right\|_{X^{\prime}}\left\|y_{n}\right\|_{Y}<\infty
$$

and

$$
T x=\sum_{n=1}^{\infty} x_{n}^{\prime}(x) y_{n}, \quad x \in X .
$$

Then we call $T: X \rightarrow Y$ a nuclear operator and if $X=Y$, then its nuclear $\operatorname{trace} \operatorname{tr}(T)$ is given by

$$
\operatorname{tr}(T)=\sum_{n=1}^{\infty} x_{n}^{\prime}\left(y_{n}\right) .
$$

It can be proved that the definition of a nuclear operator and the definition of the trace of a nuclear operator are independent of the choices of the sequences $\left\{x_{n}^{\prime}\right\}_{n=1}^{\infty}$ and $\left\{y_{n}\right\}_{n=1}^{\infty}$.

The following theorem proved by Delgado [13] present a characterization of nuclear operators in terms of the conditions on the kernel of the operator. 
Theorem 3.1. Let $\left(X_{1}, \mu_{1}\right)$ and $\left(X_{2}, \mu_{2}\right)$ be $\sigma$-finite measure spaces. Then a bounded linear operator $T: L^{p_{1}}\left(X_{1}, \mu_{1}\right) \rightarrow L^{p_{2}}\left(X_{2}, \mu_{2}\right), 1 \leq p_{1}, p_{2}<\infty$, is nuclear if and only if there exist sequences $\left\{g_{n}\right\}_{n=1}^{\infty}$ in $L^{p_{1}^{\prime}}\left(X_{1}, \mu_{1}\right)$ and $\left\{h_{n}\right\}_{n=1}^{\infty}$ in $L^{p_{2}}\left(X_{2}, \mu_{2}\right)$ such that for all $f \in L^{p_{1}}\left(X_{1}, \mu_{1}\right)$

where

$$
(T f)(x)=\int_{X_{1}} K(x, y) f(y) d \mu_{1}(y), \quad x \in X_{2},
$$

and

$$
K(x, y)=\sum_{n=1}^{\infty} h_{n}(x) g_{n}(y), \quad x \in X_{2}, y \in X_{1}
$$

$$
\sum_{n=1}^{\infty}\left\|g_{n}\right\|_{L^{p^{\prime}}\left(X_{1}, \mu_{1}\right)}\left\|h_{n}\right\|_{L^{p_{2}\left(X_{2}, \mu_{2}\right)}}<\infty .
$$

The function $K$ on $X_{2} \times X_{1}$ in Theorem 3.1 is called the kernel of the nuclear operator $T$ : $L^{p_{1}}\left(X_{1}, \mu_{1}\right) \rightarrow L^{p_{2}}\left(X_{2}, \mu_{2}\right)$.

Let $(X, \mu)$ be a $\sigma$-finite measure space. Let $T: L^{p}(X, \mu) \rightarrow L^{p}(X, \mu) 1 \leq p<\infty$, be a nuclear operator. Then by Theorem 3.1. we can find sequences $\left\{g_{n}\right\}_{n=1}^{\infty}$ in $L^{p^{\prime}}(X, \mu)$ and $\left\{h_{n}\right\}_{n=1}^{\infty}$ in $L^{p}(X, \mu)$ such that

and for all $f \in L^{p}(X, \mu)$

$$
\sum_{n=1}^{\infty}\left\|g_{n}\right\|_{L^{p^{\prime}}(X, \mu)}\left\|h_{n}\right\|_{L^{p}(X, \mu)}<\infty
$$

$$
(T f)(x)=\int_{X} K(x, y) f(y) d \mu(y), \quad x \in X,
$$

where

and it satisfies

$$
K(x, y)=\sum_{n=1}^{\infty} h_{n}(x) g_{n}(y), \quad x, y \in X
$$

$$
\int_{X}|K(x, y)| d \mu(y) \leq \sum_{n=1}^{\infty}\left\|g_{n}\right\|_{L^{p^{\prime}(X, \mu)}}\left\|h_{n}\right\|_{L^{p}(X, \mu)} .
$$

The trace $\operatorname{tr}(T)$ of $T: L^{p}(X, \mu) \rightarrow L^{p}(X, \mu)$ is given by

$$
\operatorname{tr}(T)=\int_{X} K(x, x) d \mu(x) .
$$

Now, we present a characterization of nuclear $\mathbb{Z}$-operators from $L^{p_{1}}(\mathbb{Z})$ into $L^{p_{2}}(\mathbb{Z})$. Remember that $\left\{e_{n}\right\}_{n \in \mathbb{Z}}$ be an orthonormal basis for $L^{2}(S)$ such that for every $n \in \mathbb{Z}, m\left(\left\{s \in S: e_{n}(s)=\right.\right.$ $0\})=0$.

Theorem 3.2. Let $\tau$ be a measurable function on $\mathbb{Z} \times S$. Then the $\mathbb{Z}$-operator $T_{\tau}: L^{p_{1}}(\mathbb{Z}) \rightarrow L^{p_{2}}(\mathbb{Z})$ is nuclear for $1 \leq p_{1}, p_{2}<\infty$ if and only if there exist sequences $\left\{g_{k}\right\}_{k=1}^{\infty} \in L^{p_{1}^{\prime}}(\mathbb{Z})$ and $\left\{h_{k}\right\}_{k=1}^{\infty} \in$ $L^{p_{2}}(\mathbb{Z})$ such that

and for every $n \in \mathbb{Z}$

$$
\sum_{k=-\infty}^{\infty}\left\|g_{k}\right\|_{L^{p_{1}^{\prime}(\mathbb{Z})}}\left\|h_{k}\right\|_{L^{p_{2}(\mathbb{Z})}}<\infty
$$

$$
\tau(n, s)=(m(S))^{-1 / 2}\left(\overline{e_{n}(s)}\right)^{-1} \sum_{k=-\infty}^{\infty} h_{k}(n) \overline{\left(\mathcal{F}_{\mathbb{Z}} \overline{g_{k}}\right)(s)}, \quad s \in\left\{\mathfrak{s} \in S: e_{n}(\mathfrak{s}) \neq 0\right\} .
$$


Proof. Suppose that $T_{\tau}: L^{p_{1}}(\mathbb{Z}) \rightarrow L^{p_{2}}(\mathbb{Z})$ is nuclear, where $1 \leq p_{1}, p_{2}<\infty$. Then by Theorem 3.1. there exist sequences $\left\{g_{k}\right\}_{k=1}^{\infty}$ in $L^{p_{1}^{\prime}}(\mathbb{Z})$ and $\left\{h_{k}\right\}_{k=1}^{\infty}$ in $L^{p_{2}}(\mathbb{Z})$ such that

$$
\sum_{k=1}^{\infty}\left\|g_{k}\right\|_{L^{p_{1}^{\prime}}(\mathbb{Z})}\left\|h_{k}\right\|_{L^{p_{2}(\mathbb{Z})}}<\infty
$$

and for all $f \in L^{p_{1}}(\mathbb{Z})$, we have

$$
\begin{aligned}
\left(T_{\tau} f\right)(n) & =(m(S))^{-1 / 2} \int_{S} \tau(n, s)\left(\mathcal{F}_{\mathbb{Z}} f\right)(s) \overline{e_{n}(s)} d m(s) \\
& =\int_{S} \tau(n, s)\left(\sum_{l=-\infty}^{\infty} f(l) e_{l}(s)\right) \overline{e_{n}(s)} d m(s) \\
& =\sum_{l=-\infty}^{\infty}\left(\int_{S} \tau(n, s) e_{l}(s) \overline{e_{n}(s)} d m(s)\right) f(l) \\
& =\sum_{l=-\infty}^{\infty} \sum_{k=-\infty}^{\infty} h_{k}(n) g_{k}(l) f(l) .
\end{aligned}
$$

Let $m \in \mathbb{Z}$ and the function $f$ is given by

$$
f(l)=f_{m}(l)= \begin{cases}0 & \text { if } m \neq l, \\ 1 & \text { if } m=l .\end{cases}
$$

Then from 3.2 we get

$$
\int_{S} \tau(n, s) e_{m}(s) \overline{e_{n}(s)} d m(s)=\sum_{k=-\infty}^{\infty} h_{k}(n) g_{k}(m)
$$

and so

$$
\overline{\left(\mathcal{F}_{S}\left(\overline{\tau(n, \cdot)} e_{n}(\cdot)\right)\right)(m)}=(m(S))^{-1 / 2} \sum_{k=-\infty}^{\infty} h_{k}(n) g_{k}(m) .
$$

Now,

$$
\begin{aligned}
\tau(n, s) \overline{e_{n}(s)} & =(m(S))^{1 / 2} \sum_{m=-\infty}^{\infty} \overline{\left(\mathcal{F}_{S}\left(\overline{\tau(n, \cdot)} e_{n}(\cdot)\right)\right)(m)} \overline{e_{m}(s)} \\
& =\sum_{m=-\infty}^{\infty}\left(\sum_{k=-\infty}^{\infty} h_{k}(n) g_{k}(m)\right) \overline{e_{m}(s)} \\
& =\sum_{k=-\infty}^{\infty} h_{k}(n) \sum_{m=-\infty}^{\infty} g_{k}(m) \overline{e_{m}(s)} \\
& =(m(S))^{-1 / 2} \sum_{k=-\infty}^{\infty} h_{k}(n) \overline{\left(\mathcal{F}_{\mathbb{Z}} \overline{g_{k}}\right)(s)} .
\end{aligned}
$$

Therefore, for all $n \in \mathbb{Z}$

$$
\tau(n, s)=(m(S))^{-1 / 2}\left(\overline{e_{n}(s)}\right)^{-1} \sum_{k=-\infty}^{\infty} h_{k}(n) \overline{\left(\mathcal{F}_{\mathbb{Z}} \overline{g_{k}}\right)(s)}, s \in\left\{\mathfrak{s} \in S: e_{n}(\mathfrak{s}) \neq 0\right\} .
$$


Conversely, suppose that there exist sequences $\left\{g_{k}\right\}_{k=1}^{\infty}$ in $L^{p_{1}^{\prime}}(\mathbb{Z})$ and $\left\{h_{k}\right\}_{k=1}^{\infty}$ in $L^{p_{2}}(\mathbb{Z})$ such that

$$
\sum_{k=-\infty}^{\infty}\left\|g_{k}\right\|_{L^{p_{1}^{\prime}(\mathbb{Z})}}\left\|h_{k}\right\|_{L^{p_{2}(\mathbb{Z})}}<\infty
$$

and for all $n \in \mathbb{Z}$

$$
\tau(n, s)=(m(S))^{-1 / 2}\left(\overline{e_{n}(s)}\right)^{-1} \sum_{k=-\infty}^{\infty} h_{k}(n) \overline{\left(\mathcal{F}_{\mathbb{Z}} \overline{g_{k}}\right)(s)}, \quad s \in\left\{\mathfrak{s} \in S: e_{n}(\mathfrak{s}) \neq 0\right\} .
$$

Then, for all $f \in L^{p_{1}}(\mathbb{Z})$

$$
\begin{aligned}
\left(T_{\tau} f\right)(n) & =(m(S))^{-1 / 2} \int_{S} \tau(n, s)\left(\mathcal{F}_{\mathbb{Z}} f\right)(s) \overline{e_{n}(s)} d m(s) \\
& =(m(S))^{-1} \int_{S}\left(\sum_{k=-\infty}^{\infty} h_{k}(n) \overline{\left(\mathcal{F}_{\mathbb{Z}} \overline{g_{k}}\right)(s)}\right)\left(\mathcal{F}_{\mathbb{Z} f} f(s) d m(s)\right. \\
& =(m(S))^{-1 / 2} \int_{S}\left(\sum_{k=-\infty}^{\infty} h_{k}(n) \sum_{m=-\infty}^{\infty} g_{k}(m) \overline{e_{m}(s)}\right)\left(\mathcal{F}_{\mathbb{Z}} f\right)(s) d m(s) \\
& =(m(S))^{-1 / 2} \sum_{k=-\infty}^{\infty} h_{k}(n) \sum_{m=-\infty}^{\infty} g_{k}(m) \int_{S}\left(\mathcal{F}_{\mathbb{Z}} f\right)(s) \overline{e_{m}(s)} d m(s) \\
& =\sum_{m=-\infty}^{\infty} \sum_{k=-\infty}^{\infty} h_{k}(n) g_{k}(m)\left(\mathcal{F}_{S} \mathcal{F}_{\mathbb{Z}} f\right)(m) \\
& =\sum_{m=-\infty}^{\infty} \sum_{k=-\infty}^{\infty} h_{k}(n) g_{k}(m) f(m)
\end{aligned}
$$

for all $n \in \mathbb{Z}$. Therefore by Theorem 3.1, $T_{\tau}: L^{p_{1}}(\mathbb{Z}) \rightarrow L^{p_{2}}(\mathbb{Z})$ is a nuclear operator.

In order to find the trace of a nuclear $\mathbb{Z}$-operator from $L^{p}(\mathbb{Z})$ into $L^{p}(\mathbb{Z})$, in the next theorem, we give another characterization of nuclear $\mathbb{Z}$-operator from $L^{p_{1}}(\mathbb{Z})$ into $L^{p_{2}}(\mathbb{Z}), 1 \leq p_{1}, p_{2}<\infty$.

Theorem 3.3. Let $\tau$ be a measurable function on $\mathbb{Z} \times S$. Then the $\mathbb{Z}$-operator $T_{\tau}: L^{p_{1}}(\mathbb{Z}) \rightarrow$ $L^{p_{2}}(\mathbb{Z}), 1 \leq p_{1}, p_{2}<\infty$, is nuclear if and only if there exist sequences $\left\{g_{k}\right\}_{k=1}^{\infty} \in L^{p_{1}^{\prime}}(\mathbb{Z})$ and $\left\{h_{k}\right\}_{k=1}^{\infty} \in L^{p_{2}}(\mathbb{Z})$ such that

$$
\sum_{k=-\infty}^{\infty}\left\|g_{k}\right\|_{L^{p_{1}^{\prime}(\mathbb{Z})}}\left\|h_{k}\right\|_{L^{p_{2}(\mathbb{Z})}}<\infty
$$

and

$$
\int_{S} \tau(n, s) e_{m}(s) \overline{e_{n}(s)} d m(s)=\sum_{k=-\infty}^{\infty} h_{k}(n) g_{k}(m)
$$

Proof. Let $T_{\tau}: L^{p_{1}}(\mathbb{Z}) \rightarrow L^{p_{2}}(\mathbb{Z})$ is nuclear operator. From equation 3.3 there exist sequences $\left\{g_{k}\right\}_{k=1}^{\infty} \in L^{p_{1}^{\prime}}(\mathbb{Z})$ and $\left\{h_{k}\right\}_{k=1}^{\infty} \in L^{p_{2}}(\mathbb{Z})$ such that

$$
\sum_{k=-\infty}^{\infty}\left\|g_{k}\right\|_{L^{p_{1}^{\prime}(\mathbb{Z})}}\left\|h_{k}\right\|_{L^{p_{2}(\mathbb{Z})}}<\infty
$$


and for every $n, m \in \mathbb{Z}$,

$$
\int_{S} \tau(n, s) e_{m}(s) \overline{e_{n}(s)} d m(s)=\sum_{k=-\infty}^{\infty} h_{k}(n) g_{k}(m) .
$$

Conversely, if there exist sequences $\left\{g_{k}\right\}_{k=1}^{\infty} \in L^{p_{1}^{\prime}}(\mathbb{Z})$ and $\left\{h_{k}\right\}_{k=1}^{\infty} \in L^{p_{2}}(\mathbb{Z})$ such that

$$
\sum_{k=-\infty}^{\infty}\left\|g_{k}\right\|_{L^{p_{1}^{\prime}(\mathbb{Z})}}\left\|h_{k}\right\|_{L^{p_{2}(\mathbb{Z})}}<\infty
$$

and for every $n, m \in \mathbb{Z}$,

$$
\int_{S} \tau(n, s) e_{m}(s) \overline{e_{n}(s)} d m(s)=\sum_{k=-\infty}^{\infty} h_{k}(n) g_{k}(m) .
$$

Now, for all $f \in L^{p_{1}}(\mathbb{Z})$

$$
\begin{aligned}
\left(T_{\tau} f\right)(n) & =(m(S))^{-1 / 2} \int_{S} \tau(n, s)\left(\mathcal{F}_{\mathbb{Z}} f\right)(s) \overline{e_{n}(s)} d m(s) \\
& =\int_{S} \tau(n, s) \sum_{m=-\infty}^{\infty} f(m) e_{m}(s) \overline{e_{n}(s)} d m(s) \\
& =\sum_{m=-\infty}^{\infty}\left(\int_{S} \tau(n, s) e_{m}(s) \overline{e_{n}(s)} d m(s)\right) f(m) \\
& =\sum_{m=-\infty}^{\infty} \sum_{k=1}^{\infty} h_{k}(n) g_{k}(m) f(m)
\end{aligned}
$$

for all $n \in \mathbb{Z}$. Therefore by Theorem 3.1, $T_{\tau}: L^{p_{1}}(\mathbb{Z}) \rightarrow L^{p_{2}}(\mathbb{Z})$ is a nuclear operator.

An immediate consequence of Theorem 3.3 gives the trace of a nuclear $\mathbb{Z}$-operator from $L^{p}(\mathbb{Z})$ into $L^{p}(\mathbb{Z}), 1 \leq p<\infty$. Indeed, we have the following result.

Corollary 3.4. Let $T_{\tau}: L^{p}(\mathbb{Z}) \rightarrow L^{p}(\mathbb{Z})$ be a nuclear operator for $1 \leq p<\infty$. Then the trace $\operatorname{tr}\left(T_{\tau}\right)$ of $T_{\tau}$ is given by

$$
\operatorname{tr}\left(T_{\tau}\right)=\sum_{n=-\infty}^{\infty} \int_{S} \tau(n, s)\left|e_{n}(s)\right|^{2} d m(s) .
$$

Proof. Using trace formula (3.1) and Theorem 3.3 we have

$$
\begin{aligned}
\operatorname{tr}\left(T_{\tau}\right) & =\sum_{n=-\infty}^{\infty} \sum_{k=-\infty}^{\infty} h_{k}(n) g_{k}(n) \\
& =\sum_{n=-\infty}^{\infty} \int_{S} \tau(n, s) e_{n}(s) \overline{e_{n}(s)} d m(s) \\
& =\sum_{n=-\infty}^{\infty} \int_{S} \tau(n, s)\left|e_{n}(s)\right|^{2} d m(s)
\end{aligned}
$$

If $T_{\tau}$ is a nuclear operator then as a consequence of Theorem 3.2 and Theorem 3.3 we have the following necessary conditions. 
Theorem 3.5. Let $\tau$ be a measurable function on $\mathbb{Z} \times S$ such that the coresponding $\mathbb{Z}$-operator $T_{\tau}: L^{p_{1}}(\mathbb{Z}) \rightarrow L^{p_{2}}(\mathbb{Z})$ is a nuclear operator for $2 \leq p_{1}<\infty, 1 \leq p_{2}<\infty$. Then

$$
\sum_{n=-\infty}^{\infty}\left\|\tau(n, \cdot) \overline{e_{n}(\cdot)}\right\|_{L^{p_{1}^{\prime}(S)}}^{p_{2}}<\infty
$$

Proof. Let $T_{\tau}: L^{p_{1}}(\mathbb{Z}) \rightarrow L^{p_{2}}(\mathbb{Z})$ is a nuclear operator for $2 \leq p_{1}<\infty, 1 \leq p_{2}<\infty$. By Theorem 3.2 there exist sequences $\left\{g_{k}\right\}_{k=1}^{\infty} \in L^{p_{1}^{\prime}}(\mathbb{Z})$ and $\left\{h_{k}\right\}_{k=1}^{\infty} \in L^{p_{2}}(\mathbb{Z})$ such that

$$
\sum_{k=-\infty}^{\infty}\left\|g_{k}\right\|_{L^{p_{1}^{\prime}(\mathbb{Z})}}\left\|h_{k}\right\|_{L^{p_{2}(\mathbb{Z})}}<\infty
$$

and for all $n \in \mathbb{Z}$

$$
\tau(n, s)=(m(S))^{-1 / 2}\left(\overline{e_{n}(s)}\right)^{-1} \sum_{k=-\infty}^{\infty} h_{k}(n) \overline{\left(\mathcal{F}_{\mathbb{Z}} \overline{g_{k}}\right)(s)}, s \in\left\{\mathfrak{s} \in S: e_{n}(\mathfrak{s}) \neq 0\right\}
$$

Using Minkowski's inequality and plancherel formula we get

$$
\begin{aligned}
\left\|\tau(n, \cdot) \overline{e_{n}(\cdot)}\right\|_{L^{p_{1}^{\prime}(S)}} & =\left(\int_{S}\left|\tau(n, s) \overline{e_{n}(s)}\right|^{p_{1}^{\prime}} d s\right)^{\frac{1}{p_{1}^{\prime}}} \\
& =(m(S))^{-1 / 2}\left(\int_{S}\left|\sum_{k=-\infty}^{\infty} h_{k}(n) \overline{\left(\mathcal{F}_{\mathbb{Z}} \overline{g_{k}}\right)(s)}\right|^{p_{1}^{\prime}} d s\right)^{\frac{1}{p_{1}^{\prime}}} \\
& \leq(m(S))^{-1 / 2} \sum_{k=-\infty}^{\infty}\left|h_{k}(n)\right|\left(\int_{S}\left|\overline{\left(\mathcal{F}_{\mathbb{Z}} \overline{g_{k}}\right)(s)}\right|^{p_{1}^{\prime}} d s\right)^{\frac{1}{p_{1}^{\prime}}} \\
& \leq(m(S))^{-1 / 2} \sum_{k=-\infty}^{\infty}\left|h_{k}(n)\right|\left(\int_{S}\left|\overline{\left(\mathcal{F}_{\mathbb{Z}} \overline{g_{k}}\right)(s)}\right|^{2} d s\right)^{\frac{1}{2}} \\
& =\sum_{k=-\infty}^{\infty}\left|h_{k}(n)\right|\left\|g_{k}\right\|_{L^{2}(\mathbb{Z})} \\
& \leq \sum_{k=-\infty}^{\infty}\left|h_{k}(n)\right|\left\|g_{k}\right\|_{L^{p_{1}^{\prime}}(\mathbb{Z})}
\end{aligned}
$$

and therefore

$$
\left\|\tau(n, \cdot) \overline{e_{n}(\cdot)}\right\|_{L^{p_{1}^{\prime}(S)}}^{p_{p^{2}}} \leq\left(\sum_{k=-\infty}^{\infty}\left|h_{k}(n)\right|\left\|g_{k}\right\|_{L^{p_{1}^{\prime}(\mathbb{Z})}}\right)^{p_{2}}
$$


Using Minkowski's inequality we have

$$
\begin{aligned}
\left(\sum_{n=-\infty}^{\infty}\left\|\tau(n, \cdot) \overline{e_{n}(\cdot)}\right\|_{L^{p_{1}^{\prime}(S)}}^{p_{2}}\right)^{\frac{1}{p_{2}}} & \leq\left(\sum_{n=-\infty}^{\infty}\left(\sum_{k=-\infty}^{\infty}\left|h_{k}(n)\right|\left\|g_{k}\right\|_{L^{p_{1}^{\prime}(\mathbb{Z})}}\right)^{p_{2}}\right)^{\frac{1}{p_{2}}} \\
& \leq \sum_{k=-\infty}^{\infty}\left(\sum_{n=-\infty}^{\infty}\left|h_{k}(n)\right|^{p_{2}}\right)^{\frac{1}{p_{2}}}\left\|g_{k}\right\|_{L^{p_{1}^{\prime}(\mathbb{Z})}} \\
& =\sum_{k=-\infty}^{\infty}\left\|h_{k}\right\|_{L^{p_{2}(\mathbb{Z})}}\left\|g_{k}\right\|_{L^{p_{1}^{\prime}(\mathbb{Z})}}<\infty
\end{aligned}
$$

Theorem 3.6. Let $\tau$ be a measurable function on $\mathbb{Z} \times S$ such that the corresponding $\mathbb{Z}$-operator $T_{\tau}: L^{p_{1}}(\mathbb{Z}) \rightarrow L^{p_{2}}(\mathbb{Z})$ is a nuclear operator for $1 \leq p_{1}, p_{2}<\infty$. Then

$$
\left\{\left\|\left(\mathcal{F}_{S}\left(\overline{\tau(\bullet, \cdot)} e_{\bullet}(\cdot)\right)\right)(m)\right\|_{L^{p_{2}(\mathbb{Z})}}\right\}_{m \in \mathbb{Z}} \in L^{p_{1}^{\prime}}(\mathbb{Z}) .
$$

Proof. Let $T_{\tau}: L^{p_{1}}(\mathbb{Z}) \rightarrow L^{p_{2}}(\mathbb{Z})$ is a nuclear operator for $1 \leq p_{1}, p_{2}<\infty$. Then from equation (3.4), there exist sequences $\left\{g_{k}\right\}_{k=1}^{\infty} \in L^{p_{1}^{\prime}}(\mathbb{Z})$ and $\left\{h_{k}\right\}_{k=1}^{\infty} \in L^{p_{2}}(\mathbb{Z})$ such that for all $m, n \in \mathbb{Z}$

$$
\overline{\left(\mathcal{F}_{S}\left(\overline{\tau(n, \cdot)} e_{n}(\cdot)\right)\right)(m)}=(m(S))^{-1 / 2} \sum_{k=-\infty}^{\infty} h_{k}(n) g_{k}(m)
$$

Using Minkowski's inequality we get

$$
\begin{aligned}
\left(\sum_{n=-\infty}^{\infty}\left|\left(\mathcal{F}_{S}\left(\overline{\tau(n, \cdot)} e_{n}(\cdot)\right)\right)(m)\right|^{p_{2}}\right)^{\frac{1}{p_{2}}} & =\left(\sum_{n=-\infty}^{\infty}\left|\overline{\left(\mathcal{F}_{S}\left(\overline{\tau(n, \cdot)} e_{n}(\cdot)\right)\right)(m)}\right|^{p_{2}}\right)^{\frac{1}{p_{2}}} \\
& =(m(S))^{-1 / 2}\left(\sum_{n=-\infty}^{\infty}\left|\sum_{k=-\infty}^{\infty} h_{k}(n) g_{k}(m)\right|^{p_{2}}\right)^{\frac{1}{p_{2}}} \\
& \leq(m(S))^{-1 / 2} \sum_{k=-\infty}^{\infty}\left(\sum_{n=-\infty}^{\infty}\left|h_{k}(n) g_{k}(m)\right|^{p_{2}}\right)^{\frac{1}{p_{2}}} \\
& =(m(S))^{-1 / 2} \sum_{k=-\infty}^{\infty}\left|g_{k}(m)\right|\left(\sum_{n=-\infty}^{\infty}\left|h_{k}(n)\right|^{p_{2}}\right)^{\frac{1}{p_{2}}} \\
& =(m(S))^{-1 / 2} \sum_{k=-\infty}^{\infty}\left|g_{k}(m)\right|\left\|h_{k}\right\|_{L^{p_{2}}(\mathbb{Z})}
\end{aligned}
$$


and therefore

$$
\begin{aligned}
& \left(\sum_{m=-\infty}^{\infty}\left(\sum_{n=-\infty}^{\infty}\left|\left(\mathcal{F}_{S}\left(\overline{\tau(n, \cdot)} e_{n}(\cdot)\right)\right)(m)\right|^{p_{2}}\right)^{\frac{p_{1}^{\prime}}{p_{2}}}\right)^{\frac{1}{p_{1}^{\prime}}} \\
& \leq(m(S))^{-1 / 2}\left(\sum_{m=-\infty}^{\infty}\left(\sum_{k=-\infty}^{\infty}\left|g_{k}(m)\right|\left\|h_{k}\right\|_{L^{p_{2}}(\mathbb{Z})}\right)^{p_{1}^{\prime}}\right)^{\frac{1}{p_{1}^{\prime}}} \\
& \leq(m(S))^{-1 / 2} \sum_{k=-\infty}^{\infty}\left(\sum_{n=-\infty}^{\infty}\left|g_{k}(m)\right|^{p_{1}^{\prime}}\right)^{\frac{1}{p_{1}^{\prime}}}\left\|h_{k}\right\|_{L^{p_{2}(\mathbb{Z})}} \\
& =(m(S))^{-1 / 2} \sum_{k=-\infty}^{\infty}\left\|h_{k}\right\|_{L^{p_{2}(\mathbb{Z})}\left\|g_{k}\right\|_{L^{p_{1}^{\prime}}(\mathbb{Z})}<\infty .}
\end{aligned}
$$

Theorem 3.7. Let $\tau$ be a measurable function on $\mathbb{Z} \times S$ such that the coresponding $\mathbb{Z}$-operator $T_{\tau}: L^{p_{1}}(\mathbb{Z}) \rightarrow L^{p_{2}}(\mathbb{Z})$ is a nuclear operator for $1 \leq p_{1}, p_{2}<\infty$. Then

$$
\left\{\left\|\left(\mathcal{F}_{S}\left(\overline{\tau(n, \cdot)} e_{n}(\cdot)\right)\right)(\bullet)\right\|_{L^{p_{1}^{\prime}(\mathbb{Z})}}\right\}_{n \in \mathbb{Z}} \in L^{p_{2}}(\mathbb{Z}) .
$$

Proof. Let $T_{\tau}: L^{p_{1}}(\mathbb{Z}) \rightarrow L^{p_{2}}(\mathbb{Z})$ is a nuclear operator for $1 \leq p_{1}, p_{2}<\infty$. Then from equation 3.4 , there exist sequences $\left\{g_{k}\right\}_{k=1}^{\infty} \in L^{p_{1}^{\prime}}(\mathbb{Z})$ and $\left\{h_{k}\right\}_{k=1}^{\infty} \in L^{p_{2}}(\mathbb{Z})$ such that for all $m, n \in \mathbb{Z}$

$$
\overline{\left(\mathcal{F}_{S}\left(\overline{\tau(n, \cdot)} e_{n}(\cdot)\right)\right)(m)}=(m(S))^{-1 / 2} \sum_{k=-\infty}^{\infty} h_{k}(n) g_{k}(m) .
$$

Using Minkowski's inequality we get

$$
\begin{aligned}
\left(\sum_{m=-\infty}^{\infty}\left|\left(\mathcal{F}_{S}\left(\overline{\tau(n, \cdot)} e_{n}(\cdot)\right)\right)(m)\right|^{p_{1}^{\prime}}\right)^{\frac{1}{p_{1}^{\prime}}} & =\left(\sum_{m=-\infty}^{\infty}\left|\overline{\left(\mathcal{F}_{S}\left(\overline{\tau(n, \cdot)} e_{n}(\cdot)\right)\right)(m)}\right|^{p_{1}^{\prime}}\right)^{\frac{1}{p_{1}^{\prime}}} \\
& =(m(S))^{-1 / 2}\left(\sum_{m=-\infty}^{\infty}\left|\sum_{k=-\infty}^{\infty} h_{k}(n) g_{k}(m)\right|^{p_{1}^{\prime}}\right)^{\frac{1}{p_{1}^{\prime}}} \\
& \leq(m(S))^{-1 / 2} \sum_{k=-\infty}^{\infty}\left(\sum_{m=-\infty}^{\infty}\left|h_{k}(n) g_{k}(m)\right|^{p_{1}^{\prime}}\right)^{\frac{1}{p_{1}^{\prime}}} \\
& =(m(S))^{-1 / 2} \sum_{k=-\infty}^{\infty}\left|h_{k}(n)\right|\left(\sum_{m=-\infty}^{\infty}\left|g_{k}(m)\right|^{p_{1}^{\prime}}\right)^{\frac{1}{p_{1}^{\prime}}} \\
& =(m(S))^{-1 / 2} \sum_{k=-\infty}^{\infty}\left|h_{k}(n)\right|\left\|g_{k}\right\|_{L^{p_{1}^{\prime}}(\mathbb{Z})}
\end{aligned}
$$


and therefore

$$
\begin{aligned}
& \left(\sum_{n=-\infty}^{\infty}\left(\sum_{m=-\infty}^{\infty}\left|\left(\mathcal{F}_{S}\left(\overline{\tau(n, \cdot)} e_{n}(\cdot)\right)\right)(m)\right|^{p_{1}^{\prime}}\right)^{\frac{p_{2}}{p_{1}^{\prime}}}\right)^{\frac{1}{p_{2}}} \\
& \leq(m(S))^{-1 / 2}\left(\sum_{n=-\infty}^{\infty}\left(\sum_{k=-\infty}^{\infty}\left|h_{k}(n)\right|\left\|g_{k}\right\|_{L^{p_{1}^{\prime}}(\mathbb{Z})}\right)^{p_{2}}\right)^{\frac{1}{p_{2}}} \\
& \leq(m(S))^{-1 / 2} \sum_{k=-\infty}^{\infty}\left(\sum_{n=-\infty}^{\infty}\left|h_{k}(n)\right|^{p_{2}}\right)^{\frac{1}{p_{2}}}\left\|g_{k}\right\|_{L^{p_{1}^{\prime}}(\mathbb{Z})} \\
& =(m(S))^{-1 / 2} \sum_{k=-\infty}^{\infty}\left\|h_{k}\right\|_{L^{p_{2}(\mathbb{Z})}\left\|g_{k}\right\|_{L^{p_{1}^{\prime}}(\mathbb{Z})}<\infty .}
\end{aligned}
$$

3.1. Adjoints. Let $T_{\tau}: L^{p_{1}}(\mathbb{Z}) \rightarrow L^{p_{2}}(\mathbb{Z}), 1 \leq p_{1}, p_{2}<\infty$, be a nuclear operator. In this sub-section we show that the adjoint operator $T_{\tau}^{*}: L^{p_{2}^{\prime}}(\mathbb{Z}) \rightarrow L^{p_{1}^{\prime}}(\mathbb{Z})$ of $T_{\tau}$, is also nuclear. We also present a necessary and sufficient condition on the symbol $\tau$ so that the corresponding nuclear operator $T_{\tau}$ from $L^{2}(\mathbb{Z})$ into $L^{2}(\mathbb{Z})$ to be self-adjoint.

Theorem 3.8. Let $\tau$ be a measurable function on $\mathbb{Z} \times S$ such that $T_{\tau}: L^{p_{1}}(\mathbb{Z}) \rightarrow L^{p_{2}}(\mathbb{Z})$ is a nuclear operator for $1 \leq p_{1}, p_{2}<\infty$. Then $T_{\tau}^{*}$, the adjoint of $T_{\tau}$, is also a nuclear operator from $L^{p_{2}^{\prime}}(\mathbb{Z})$ into $L^{p_{1}^{\prime}}(\mathbb{Z})$ with symbol $\tau^{*}$ given by

$$
\tau^{*}(n, s)=(m(S))^{-1 / 2}\left(\overline{e_{n}(s)}\right)^{-1} \sum_{k=-\infty}^{\infty} \overline{g_{k}(n)\left(\mathcal{F}_{\mathbb{Z}} h_{k}\right)(s)}, \quad(n, s) \in \mathbb{Z} \times\left\{\mathfrak{s} \in S: e_{n}(\mathfrak{s}) \neq 0\right\},
$$

where $\left\{g_{k}\right\}_{k=1}^{\infty}$ and $\left\{h_{k}\right\}_{k=1}^{\infty}$ are two sequences in $L^{p_{1}^{\prime}}(\mathbb{Z})$ and $L^{p_{2}}(\mathbb{Z})$ respectively such that

$$
\sum_{k=-\infty}^{\infty}\left\|g_{k}\right\|_{L^{p_{1}^{\prime}(\mathbb{Z})}}\left\|h_{k}\right\|_{L^{p_{2}(\mathbb{Z})}}<\infty
$$

Proof. For all $f \in L^{p_{1}}(\mathbb{Z})$ and $g \in L^{p_{2}}(\mathbb{Z})$, we have

$$
\sum_{i=-\infty}^{\infty}\left(T_{\tau} f\right)(i) \overline{g(i)}=\sum_{i=-\infty}^{\infty} f(i) \overline{T_{\tau}^{*} g(i)}
$$

Therefore,

$$
\left.\sum_{i=-\infty}^{\infty}\left(\int_{S} \tau(i, s)\left(\mathcal{F}_{\mathbb{Z}} f\right)(s) \overline{e_{i}(s)} d m(s)\right) \overline{g(i)}=\sum_{i=-\infty}^{\infty} f(i) \overline{\left(\int_{S} \tau^{*}(i, s)\left(\mathcal{F}_{\mathbb{Z}} g\right)(s) \overline{e_{i}(s)}\right.} d m(s)\right) .
$$

Now, let $m, n \in \mathbb{Z}$ and for all $i \in \mathbb{Z}$,

$$
f(i)=f_{n}(i)=\left\{\begin{array}{ll}
0 & \text { if } i \neq n, \\
1 & \text { if } i=n,
\end{array} \quad g(i)=g_{m}(i)= \begin{cases}0 & \text { if } i \neq m \\
1 & \text { if } i=m .\end{cases}\right.
$$


Therefore from 3.5 we have,

$$
\int_{S} \tau(m, s) e_{n}(s) \overline{e_{m}(s)} d m(s)=\overline{\int_{S} \tau^{*}(n, s) e_{m}(s) \overline{e_{n}(s)} d m(s)}
$$

and thus

$$
\overline{\left(\mathcal{F}_{S}\left(\overline{\tau(m, \cdot)} e_{m}(\cdot)\right)\right)(n)}=\left(\mathcal{F}_{S}\left(\overline{\tau^{*}(n, \cdot)} e_{n}(\cdot)\right)\right)(m) .
$$

Since $T_{\tau}: L^{p_{1}}(\mathbb{Z}) \rightarrow L^{p_{2}}(\mathbb{Z})$ is a nuclear, from Theorem 3.2 there exist sequences $\left\{g_{k}\right\}_{k=1}^{\infty} \in L^{p_{1}^{\prime}}(\mathbb{Z})$ and $\left\{h_{k}\right\}_{k=1}^{\infty} \in L^{p_{2}}(\mathbb{Z})$ such that

$$
\sum_{k=-\infty}^{\infty}\left\|g_{k}\right\|_{L^{p_{1}^{\prime}(\mathbb{Z})}}\left\|h_{k}\right\|_{L^{p_{2}(\mathbb{Z})}}<\infty
$$

and for all $n \in \mathbb{Z}$

$$
\tau(n, s)=(m(S))^{-1 / 2}\left(\overline{e_{n}(s)}\right)^{-1} \sum_{k=-\infty}^{\infty} h_{k}(n) \overline{\left(\mathcal{F}_{\mathbb{Z}} \overline{g_{k}}\right)(s)}, s \in\left\{\mathfrak{s} \in S: e_{n}(\mathfrak{s}) \neq 0\right\} .
$$

Using (3.6) and the nuclearity of $T_{\tau}$, we have

$$
\begin{aligned}
\overline{\tau^{*}(n, s)} e_{n}(s) & =(m(S))^{1 / 2} \sum_{m=-\infty}^{\infty}\left(\mathcal{F}_{S}\left(\overline{\tau^{*}(n, \cdot)} e_{n}(\cdot)\right)\right)(m) e_{m}(s) \\
& =(m(S))^{1 / 2} \sum_{m=-\infty}^{\infty} \overline{\left(\mathcal{F}_{S}\left(\overline{\tau(m, \cdot)} e_{m}(\cdot)\right)\right)(n)} e_{m}(s) \\
& =\sum_{m=-\infty}^{\infty}\left(\int_{S} \tau(m, w) \overline{e_{m}(w)} e_{n}(w) d m(w)\right) e_{m}(s) \\
& =(m(S))^{-1 / 2} \sum_{m=-\infty}^{\infty}\left(\int_{S}\left(\overline{\sum_{k=-\infty}} h_{k}(m) \overline{\left(\mathcal{F}_{\mathbb{Z}} \overline{g_{k}}\right)(w)}\right) e_{n}(w) d m(w)\right) e_{m}(s) \\
& =(m(S))^{-1 / 2} \sum_{m=-\infty}^{\infty}\left(\overline{\sum_{k=-\infty}^{\infty} h_{k}(m)\left(\int_{S}\left(\mathcal{F}_{\mathbb{Z}} \overline{g_{k}}\right)(w) \overline{e_{n}(w)} d m(w)\right)}\right) e_{m}(s) \\
& =\sum_{m=-\infty}^{\infty}\left(\sum_{k=-\infty}^{\infty} h_{k}(m) \overline{\left(\mathcal{F}_{S} \mathcal{F}_{\mathbb{Z}} \overline{g_{k}}\right)(n)}\right) e_{m}(s) \\
& =\sum_{m=-\infty}^{\infty} \sum_{k=-\infty}^{\infty} h_{k}(m) g_{k}(n) e_{m}(s) \\
& =\sum_{k=-\infty}^{\infty} g_{k}(n) \sum_{m=-\infty}^{\infty} h_{k}(m) e_{m}(s) \\
& (m(S))^{-1 / 2} \sum_{k=-\infty}^{\infty} g_{k}(n)\left(\mathcal{F}_{\mathbb{Z}} h_{k}\right)(s) .
\end{aligned}
$$

Therefore, for all $n \in \mathbb{Z}$

$$
\tau^{*}(n, s)=(m(S))^{-1 / 2}\left(\overline{e_{n}(s)}\right)^{-1} \sum_{k=-\infty}^{\infty} \overline{g_{k}(n)\left(\mathcal{F}_{\mathbb{Z}} h_{k}\right)(s)}, s \in\left\{\mathfrak{s} \in S: e_{n}(\mathfrak{s}) \neq 0\right\} .
$$


As a consequence of the above theorem and the help of Theorem 3.2. we can give a criterion in terms of symbol such that the corresponding $\mathbb{Z}$-operator to be self-adjoint.

Corollary 3.9. Let $\tau$ be a measurable function on $\mathbb{Z} \times S$ such that $T_{\tau}: L^{2}(\mathbb{Z}) \rightarrow L^{2}(\mathbb{Z})$ is nuclear. Then $T_{\tau}: L^{2}(\mathbb{Z}) \rightarrow L^{2}(\mathbb{Z})$ is self-adjoint if and only if there exist sequences $\left\{g_{k}\right\}_{k=1}^{\infty}$ and $\left\{h_{k}\right\}_{k=1}^{\infty}$ in $L^{2}(\mathbb{Z})$ such that

$$
\begin{gathered}
\sum_{k=-\infty}^{\infty}\left\|h_{k}\right\|_{L^{2}(\mathbb{Z})}\left\|g_{k}\right\|_{L^{2}(\mathbb{Z})}<\infty, \\
\sum_{k=-\infty}^{\infty} h_{k}(n) \overline{\left(\mathcal{F}_{\mathbb{Z}} \overline{g_{k}}\right)(s)}=\sum_{k=-\infty}^{\infty} \overline{g_{k}(n)\left(\mathcal{F}_{\mathbb{Z}} h_{k}\right)(s)},
\end{gathered}
$$

and

$$
\tau(n, s) \overline{e_{n}(s)}=(m(S))^{-1 / 2} \sum_{k=-\infty}^{\infty} h_{k}(n) \overline{\left(\mathcal{F}_{\mathbb{Z}} \overline{g_{k}}\right)(s)},
$$

for all $n \in \mathbb{Z}, s \in\left\{\mathfrak{s} \in S: e_{n}(\mathfrak{s}) \neq 0\right\}$.

We can give another formula for the adjoints of nuclear $\mathbb{Z}$-operators in terms of symbols. Indeed, we have the following theorem.

Theorem 3.10. Let $\tau$ be a measurable function on $\mathbb{Z} \times S$ such that the coresponding $\mathbb{Z}$-operator $T_{\tau}: L^{p_{1}}(\mathbb{Z}) \rightarrow L^{p_{2}}(\mathbb{Z})$ is a nuclear operator for $1 \leq p_{1}, p_{2}<\infty$. Then for all $m \in \mathbb{Z}$ and $w \in\{\mathfrak{s} \in$ $\left.S: e_{m}(\mathfrak{s}) \neq 0\right\}$,

$$
\tau^{*}(m, w)=m(S)\left(\overline{e_{m}(w)}\right)^{-1} \sum_{n=-\infty}^{\infty} \overline{e_{n}(w)} \int_{S} \overline{e_{m}(s) \tau(n, s)} e_{n}(s) d m(s) .
$$

Proof. Let $T_{\tau}: L^{p_{1}}(\mathbb{Z}) \rightarrow L^{p_{2}}(\mathbb{Z})$ is a nuclear operator for $1 \leq p_{1}, p_{2}<\infty$. By Theorem 3.2 , there exist sequences $\left\{g_{k}\right\}_{k=1}^{\infty} \in L^{p_{1}^{\prime}}(\mathbb{Z})$ and $\left\{h_{k}\right\}_{k=1}^{\infty} \in L^{p_{2}}(\mathbb{Z})$ such that

$$
\sum_{k=-\infty}^{\infty}\left\|g_{k}\right\|_{L^{p_{1}^{\prime}(\mathbb{Z})}}\left\|h_{k}\right\|_{L^{p_{2}(\mathbb{Z})}}<\infty
$$

and for all $(n, s) \in \mathbb{Z} \times\left\{\mathfrak{s} \in S: e_{n}(\mathfrak{s}) \neq 0\right\}$

$$
\tau(n, s)=(m(S))^{-1 / 2}\left(\overline{e_{n}(s)}\right)^{-1} \sum_{k=-\infty}^{\infty} h_{k}(n) \overline{\mathcal{F}_{\mathbb{Z}} \overline{g_{k}}(s)} .
$$

Let $m \in \mathbb{Z}$. Then

$$
\overline{e_{m}(s) \tau(n, s)} e_{n}(s)=(m(S))^{-1 / 2} \overline{e_{m}(s)} \sum_{k=-\infty}^{\infty} \overline{h_{k}(n)} \mathcal{F}_{\mathbb{Z}} \overline{g_{k}}(s)
$$


and

$$
\begin{aligned}
\int_{S} \overline{e_{m}(s) \tau(n, s)} e_{n}(s) d m(s) & =(m(S))^{-1 / 2} \sum_{k=-\infty}^{\infty} \overline{h_{k}(n)} \int_{S} \mathcal{F}_{\mathbb{Z}} \overline{g_{k}}(s) \overline{e_{m}(s)} d m(s) \\
& =\sum_{k=-\infty}^{\infty} \overline{h_{k}(n)} \overline{g_{k}(m)} .
\end{aligned}
$$

Thus, using Theorem 3.8 we get

$$
\begin{aligned}
& m(S) \sum_{n=-\infty}^{\infty} \overline{e_{n}(w)} \int_{S} \overline{e_{m}(s) \tau(n, s)} e_{n}(s) d m(s) \\
& =m(S) \sum_{n=-\infty}^{\infty} \overline{e_{n}(w)} \sum_{k=-\infty}^{\infty} \overline{h_{k}(n)} \overline{g_{k}(m)} \\
& =m(S) \sum_{k=-\infty}^{\infty}\left(\sum_{n=-\infty}^{\infty} \overline{e_{n}(w) h_{k}(n)}\right) \overline{g_{k}(m)} \\
& =(m(S))^{1 / 2} \sum_{k=-\infty}^{\infty} \overline{\left(\mathcal{F}_{\mathbb{Z}} h_{k}\right)(w) g_{k}(m)}=\tau^{*}(m, w) \overline{e_{m}(w)} .
\end{aligned}
$$

Therefore for all $m \in \mathbb{Z}$

$$
\tau^{*}(m, w)=m(S)\left(\overline{e_{m}(w)}\right)^{-1} \sum_{n=-\infty}^{\infty} \overline{e_{n}(w)} \int_{S} \overline{e_{m}(s) \tau(n, s)} e_{n}(s) d m(s), w \in\left\{\mathfrak{s} \in S: e_{m}(\mathfrak{s}) \neq 0\right\} .
$$

3.2. Products. In the next subsection we show that the product of a nuclear $\mathbb{Z}$-operator with a bounded operator is again a nuclear operator and as a consequence, we give a necessary and sufficient condition on symbol so that the corresponding nuclear $\mathbb{Z}$-operator to be normal.

Theorem 3.11. Let $\tau$ and $\eta$ be two measurable function on $\mathbb{Z} \times S$ such that the corresponding $\mathbb{Z}$-operators $T_{\tau}: L^{p}(\mathbb{Z}) \rightarrow L^{p}(\mathbb{Z})$ and $T_{\eta}: L^{p}(\mathbb{Z}) \rightarrow L^{p}(\mathbb{Z})$ are nuclear operator and bounded operator respectively for $1 \leq p<\infty$. Then $T_{\eta} T_{\tau}: L^{p}(\mathbb{Z}) \rightarrow L^{p}(\mathbb{Z})$ is a nuclear operator $T_{\lambda}: L^{p}(\mathbb{Z}) \rightarrow L^{p}(\mathbb{Z})$ with symbol $\lambda$ is given by

$$
\lambda(m, s)=(m(S))^{1 / 2}\left(\overline{e_{m}(s)}\right)^{-1} \sum_{l=-\infty}^{\infty} \tau(l, s) \overline{e_{l}(s)}\left(\mathcal{F}_{S}\left(\overline{\eta^{*}(l, \cdot)} e_{l}(\cdot)\right)\right)(m),
$$

for all $m \in \mathbb{Z}, s \in\left\{\mathfrak{s} \in S: e_{m}(\mathfrak{s}) \neq 0\right\}$.

Proof. For all $f, g \in L^{p}(\mathbb{Z})$, we have

$$
\sum_{l=-\infty}^{\infty}\left(T_{\lambda} f\right)(l) \overline{g(l)}=\sum_{l=-\infty}^{\infty}\left(T_{\eta} T_{\tau} f\right)(l) \overline{g(l)}=\sum_{l=-\infty}^{\infty}\left(T_{\tau} f\right)(l) \overline{T_{\eta^{*}} g(l)} .
$$


Therefore,

$$
\begin{aligned}
& \sum_{l=-\infty}^{\infty}\left(\int_{S} \lambda(l, s)\left(\mathcal{F}_{\mathbb{Z}} f\right)(s) \overline{e_{l}(s)} d m(s)\right) \overline{g(l)} \\
= & \sum_{l=-\infty}^{\infty}\left(\int_{S} \tau(l, s)\left(\mathcal{F}_{\mathbb{Z}} f\right)(s) \overline{e_{l}(s)} d m(s)\right) \overline{\left(T_{\eta^{*}} g\right)(l)} \\
= & (m(S))^{-1 / 2} \sum_{l=-\infty}^{\infty}\left(\int_{S} \tau(l, s)\left(\mathcal{F}_{\mathbb{Z}} f\right)(s) \overline{e_{l}(s)} d m(s)\right)\left(\overline{\int_{S} \eta^{*}(l, w)\left(\mathcal{F}_{\mathbb{Z}} g\right)(w) \overline{e_{l}(w)} d m(w)}\right)
\end{aligned}
$$

Now, let $m, n \in \mathbb{Z}$ and for all $l \in \mathbb{Z}$,

$$
f(l)=f_{n}(l)=\left\{\begin{array}{ll}
0 & \text { if } l \neq n, \\
1 & \text { if } l=n,
\end{array} \quad g(l)=g_{m}(l)= \begin{cases}0 & \text { if } l \neq m \\
1 & \text { if } l=m\end{cases}\right.
$$

Therefore from 3.7 we have,

$$
\begin{aligned}
& \int_{S} \lambda(m, s) e_{n}(s) \overline{e_{m}(s)} d m(s) \\
& =\sum_{l=-\infty}^{\infty}\left(\int_{S} \tau(l, s) e_{n}(s) \overline{e_{l}(s)} d m(s)\right) \overline{\int_{S} \eta^{*}(l, w) e_{m}(w) \overline{e_{l}(w)}} d m(w)
\end{aligned}
$$

and

$$
\overline{\left(\mathcal{F}_{S}\left(\overline{\lambda(m, \cdot)} e_{m}(\cdot)\right)\right)(n)}=(m(S))^{1 / 2} \sum_{l=-\infty}^{\infty} \overline{\left(\mathcal{F}_{S}\left(\overline{\tau(l, \cdot)} e_{l}(\cdot)\right)\right)(n)}\left(\mathcal{F}_{S}\left(\overline{\eta^{*}(l, \cdot)} e_{l}(\cdot)\right)\right)(m)
$$

So,

$$
\begin{aligned}
\lambda(m, s) \overline{e_{m}(s)} & =(m(S))^{1 / 2} \sum_{n=-\infty}^{\infty} \overline{\left(\mathcal{F}_{S}\left(\overline{\lambda(m, \cdot)} e_{m}(\cdot)\right)\right)(n)} \overline{e_{n}(s)} \\
& =m(S) \sum_{n=-\infty}^{\infty} \sum_{l=-\infty}^{\infty} \overline{\left(\mathcal{F}_{S}\left(\overline{\tau(l, \cdot)} e_{l}(\cdot)\right)\right)(n)}\left(\mathcal{F}_{S}\left(\overline{\eta^{*}(l, \cdot)} e_{l}(\cdot)\right)\right)(m) \overline{e_{n}(s)} \\
& =m(S) \sum_{l=-\infty}^{\infty}\left(\mathcal{F}_{S}\left(\overline{\eta^{*}(l, \cdot)} e_{l}(\cdot)\right)\right)(m) \sum_{n=-\infty}^{\infty} \overline{\left(\mathcal{F}_{S}\left(\overline{\tau(l, \cdot)} e_{l}(\cdot)\right)\right)(n)} \overline{e_{n}(s)} \\
& =(m(S))^{1 / 2} \sum_{l=-\infty}^{\infty} \tau(l, s) \overline{e_{l}(s)}\left(\mathcal{F}_{S}\left(\overline{\eta^{*}(l, \cdot)} e_{l}(\cdot)\right)\right)(m) .
\end{aligned}
$$

Thus, for all $m \in \mathbb{Z}$

$$
\lambda(m, s)=(m(S))^{1 / 2}\left(\overline{e_{m}(s)}\right)^{-1} \sum_{l=-\infty}^{\infty} \tau(l, s) \overline{e_{l}(s)}\left(\mathcal{F}_{S}\left(\overline{\eta^{*}(l, \cdot)} e_{l}(\cdot)\right)\right)(m), s \in\left\{\mathfrak{s} \in S: e_{m}(\mathfrak{s}) \neq 0\right\} .
$$

Since $T_{\tau}$ is nuclear operator and $T_{\eta}$ is bounded operator, by applying Theorem 3.2 , the proof will be complete.

As a consequence of the above theorem, we can give a criterion in terms of symbol such that the corresponding $\mathbb{Z}$-operator to be normal. 
Corollary 3.12. Let $\tau$ and $\eta$ be two measurable function on $\mathbb{Z} \times S$ such that the corresponding $\mathbb{Z}$-operators $T_{\tau}: L^{p}(\mathbb{Z}) \rightarrow L^{p}(\mathbb{Z})$ is bounded and $T_{\eta}: L^{p}(\mathbb{Z}) \rightarrow L^{p}(\mathbb{Z})$ is a nuclear operator for $1 \leq p<\infty$. Then $T_{\tau} T_{\eta}: L^{p}(\mathbb{Z}) \rightarrow L^{p}(\mathbb{Z})$ is a nuclear operator $T_{\lambda}: L^{p}(\mathbb{Z}) \rightarrow L^{p}(\mathbb{Z})$ with symbol $\lambda$ is given by, for all $m \in \mathbb{Z}, s \in\left\{\mathfrak{s} \in S: e_{m}(\mathfrak{s}) \neq 0\right\}$

$$
\lambda(m, s)=(m(S))^{1 / 2}\left(\overline{e_{m}(s)}\right)^{-1} \sum_{n=-\infty}^{\infty} \eta(n, s) \overline{e_{n}(s)}\left(\mathcal{F}_{S}\left(\overline{\tau^{*}(n, \cdot)} e_{n}(\cdot)\right)\right)(m),
$$

Corollary 3.13. Let $\tau$ be a function on $\mathbb{Z} \times S$ such that $T_{\tau}: L^{2}(\mathbb{Z}) \rightarrow L^{2}(\mathbb{Z})$ is a nuclear operator. Then $T_{\tau}: L^{2}(\mathbb{Z}) \rightarrow L^{2}(\mathbb{Z})$ is normal if and only if for all $m \in \mathbb{Z}, s \in\left\{\mathfrak{s} \in S: e_{m}(\mathfrak{s}) \neq 0\right\}$,

$$
\sum_{n=-\infty}^{\infty} \tau(n, s) \overline{e_{n}(s)}\left(\mathcal{F}_{S}\left(\overline{\tau^{*}(n, \cdot)} e_{n}(\cdot)\right)\right)(m)=\sum_{n=-\infty}^{\infty} \tau^{*}(n, s) \overline{e_{n}(s)}\left(\mathcal{F}_{S}\left(\overline{\tau(n, \cdot)} e_{n}(\cdot)\right)\right)(m) .
$$

\section{S-Operators}

In this section, we study the nuclearity property of $S$-operator related to the finite measure space $(S, \mathcal{B}, m)$. We begin this section by presenting a sufficient and necessary conditions on symbol such that the corresponding $S$-operator from $L^{p_{1}}(S)$ into $L^{p_{2}}(S)$ is nuclear for $1 \leq p_{1}, p_{2}<\infty$.

Theorem 4.1. Let $\sigma$ be a measurable function on $S \times \mathbb{Z}$. Then the $S$-operator $T_{\sigma}: L^{p_{1}}(S) \rightarrow L^{p_{2}}(S)$ is nuclear for $1 \leq p_{1}, p_{2}<\infty$ if and only if there exist sequences $\left\{g_{k}\right\}_{k=1}^{\infty} \in L^{p_{1}^{\prime}}(S)$ and $\left\{h_{k}\right\}_{k=1}^{\infty} \in$ $L^{p_{2}}(S)$ such that

$$
\sum_{k=-\infty}^{\infty}\left\|g_{k}\right\|_{L^{p_{1}^{\prime}(S)}}\left\|h_{k}\right\|_{L^{p_{2}(S)}}<\infty
$$

and for every $n \in \mathbb{Z}$

$$
\sigma(s, n)=(m(S))^{1 / 2}\left(e_{n}(s)\right)^{-1} \sum_{k=-\infty}^{\infty} h_{k}(s) \overline{\left(\mathcal{F}_{S} \overline{g_{k}}\right)(n)}, s \in\left\{\mathfrak{s} \in S: e_{n}(\mathfrak{s}) \neq 0\right\} .
$$

Proof. Suppose that $T_{\sigma}: L^{p_{1}}(S) \rightarrow L^{p_{2}}(S)$ is nuclear for $1 \leq p_{1}, p_{2}<\infty$. Then by Theorem 3.1 . there exist sequences $\left\{g_{k}\right\}_{k=1}^{\infty}$ in $L^{p_{1}^{\prime}}(S)$ and $\left\{h_{k}\right\}_{k=1}^{\infty}$ in $L^{p_{2}}(S)$ such that

$$
\sum_{k=1}^{\infty}\left\|g_{k}\right\|_{L^{p_{1}^{\prime}(S)}}\left\|h_{k}\right\|_{L^{p_{2}(S)}}<\infty
$$

and for all $f \in L^{p_{1}}(S)$ we have

$$
\left(T_{\sigma} f\right)(s)=\int_{S}\left(\sum_{k=-\infty}^{\infty} h_{k}(s) g_{k}(w)\right) f(w) d m(w) .
$$

Now, using the definition of $S$-operator $T_{\sigma}$, that is

$$
\begin{aligned}
\left(T_{\sigma} f\right)(s) & =(m(S))^{1 / 2} \sum_{k=-\infty}^{\infty} \sigma(s, k)\left(\mathcal{F}_{S} f\right)(k) e_{k}(s) \\
& =\int_{S}\left(\sum_{k=-\infty}^{\infty} \sigma(s, k) e_{k}(s) \overline{e_{k}(w)}\right) f(w) d m(w),
\end{aligned}
$$


we get

$$
\int_{S}\left(\sum_{k=-\infty}^{\infty} \sigma(s, k) e_{k}(s) \overline{e_{k}(w)}\right) f(w) d m(w)=\int_{S}\left(\sum_{k=-\infty}^{\infty} h_{k}(s) g_{k}(w)\right) f(w) d m(w)
$$

for all $s \in S$. Let $f=e_{n}$. Since

$$
\int_{S} e_{n}(w) \overline{e_{k}(w)} d m(w)= \begin{cases}0, & k \neq n \\ 1, & k=n\end{cases}
$$

it follows from (4.1) that

$$
\begin{aligned}
\sigma(s, n) e_{n}(s) & =\int_{S}\left(\sum_{k=-\infty}^{\infty} \sigma(s, k) e_{k}(s) \overline{e_{k}(w)}\right) e_{n}(w) d m(w) \\
& =\sum_{k=-\infty}^{\infty} h_{k}(s) \int_{S} g_{k}(w) e_{n}(w) d m(w) \\
& =(m(S))^{1 / 2} \sum_{k=1}^{\infty} h_{k}(s) \overline{\left(\mathcal{F}_{S} \overline{g_{k}}\right)(n)} .
\end{aligned}
$$

Thus, for every $n \in \mathbb{Z}$

$$
\sigma(s, n)=(m(S))^{1 / 2}\left(e_{n}(s)\right)^{-1} \sum_{k=1}^{\infty} h_{k}(s) \overline{\left(\mathcal{F}_{S} \overline{g_{k}}\right)(n)}, s \in\left\{\mathfrak{s} \in S: e_{n}(\mathfrak{s}) \neq 0\right\} .
$$

Conversely, suppose that there exist sequences $\left\{g_{k}\right\}_{k=1}^{\infty}$ in $L^{p_{1}^{\prime}}(S)$ and $\left\{h_{k}\right\}_{k=1}^{\infty}$ in $L^{p_{2}}(S)$ such that

$$
\sum_{k=-\infty}^{\infty}\left\|g_{k}\right\|_{L^{p_{1}^{\prime}(S)}}\left\|h_{k}\right\|_{L^{p_{2}(S)}}<\infty
$$

and for every $n \in \mathbb{Z}$

$$
\sigma(s, n)=(m(S))^{1 / 2}\left(e_{n}(s)\right)^{-1} \sum_{k=-\infty}^{\infty} h_{k}(s) \overline{\left(\mathcal{F}_{S} \overline{g_{k}}\right)(n)}, s \in\left\{\mathfrak{s} \in S: e_{n}(\mathfrak{s}) \neq 0\right\} .
$$

Then, for all $f \in L^{p_{1}}(S)$

$$
\begin{aligned}
\left(T_{\sigma} f\right)(s) & =(m(S))^{1 / 2} \sum_{n=-\infty}^{\infty} \sigma(s, n)\left(\mathcal{F}_{S} f\right)(n) e_{n}(s) \\
& =m(S) \sum_{n=-\infty}^{\infty}\left(\sum_{k=-\infty}^{\infty} h_{k}(s) \overline{\mathcal{F}_{S} \overline{g_{k}}(n)}\right)\left(\mathcal{F}_{S} f\right)(n) \\
& =(m(S))^{1 / 2} \sum_{n=-\infty}^{\infty}\left(\sum_{k=-\infty}^{\infty} h_{k}(s) \int_{S} g_{k}(w) e_{n}(w) d m(w)\right)\left(\mathcal{F}_{S} f\right)(n) \\
& =(m(S))^{1 / 2} \int_{S} \sum_{n=-\infty}^{\infty}\left(\mathcal{F}_{S} f\right)(n) e_{n}(w) \sum_{k=-\infty}^{\infty} h_{k}(s) g_{k}(w) d m(w) \\
& =\int_{S}\left(\sum_{k=-\infty}^{\infty} h_{k}(s) g_{k}(w)\right)\left(\mathcal{F}_{\mathbb{Z}} \mathcal{F}_{S} f\right)(w) d m(w)
\end{aligned}
$$




$$
=\int_{S}\left(\sum_{k=-\infty}^{\infty} h_{k}(s) g_{k}(w)\right) f(w) d m(w)
$$

for all $s \in S$. Therefore, by Theorem 3.1. $T_{\sigma}: L^{p_{1}}(S) \rightarrow L^{p_{2}}(S)$ is a nuclear operator.

4.1. Product. The following theorem give a symbolic characterization of product of a nuclear $S$ operator with a bounded $S$-operator.

Theorem 4.2. Let $\sigma$ and $\eta$ be two measurable function on $S \times \mathbb{Z}$ such that $T_{\sigma}: L^{p}(S) \rightarrow L^{p}(S)$ is a nuclear operator and $T_{\eta}: L^{p}(S) \rightarrow L^{p}(S)$ is a bounded linear operator for $1 \leq p<\infty$. Then $T_{\eta} T_{\sigma}: L^{p}(S) \rightarrow L^{p}(S)$ is a nuclear operator $T_{\lambda}: L^{p}(S) \rightarrow L^{p}(S)$ with symbol $\lambda$ is given by

$$
\lambda(s, m)=(m(S))^{1 / 2}\left(e_{m}(s)\right)^{-1} \sum_{k=-\infty}^{\infty} h_{k}^{\prime}(s) \overline{\left(\mathcal{F}_{S} \overline{g_{k}}\right)(m)}, n \in \mathbb{Z}, s \in\left\{\mathfrak{s} \in S: e_{n}(\mathfrak{s}) \neq 0\right\}
$$

where

$$
h_{k}^{\prime}(s)=(m(S))^{1 / 2} \sum_{n=-\infty}^{\infty} \eta(s, n) e_{n}(s)\left(\mathcal{F}_{S} h_{k}\right)(n), s \in\left\{\mathfrak{s} \in S: e_{n}(\mathfrak{s}) \neq 0\right\}
$$

Proof. Let $f \in L^{p}(S)$. Then for all $s \in S$,

$$
\begin{aligned}
\left(T_{\eta} T_{\sigma} f\right)(s) & =(m(S))^{1 / 2} \sum_{n=-\infty}^{\infty} \eta(s, n)\left(\mathcal{F}_{S} T_{\sigma} f\right)(n) e_{n}(s) \\
& =\sum_{n=-\infty}^{\infty} \eta(s, n) e_{n}(s)\left(\int_{S} T_{\sigma} f(w) \overline{e_{n}(w)} d m(w)\right) \\
& =(m(S))^{1 / 2} \sum_{n=-\infty}^{\infty} \eta(s, n) e_{n}(s) \int_{S} \overline{e_{n}(w)}\left(\sum_{m=-\infty}^{\infty} \sigma(w, m)\left(\mathcal{F}_{S} f\right)(m) e_{m}(w)\right) d m(w)
\end{aligned}
$$

Since $T_{\sigma}: L^{p}(S) \rightarrow L^{p}(S)$ is a nuclear operator, by theorem 4.1, there exist sequences $\left\{g_{k}\right\}_{k=1}^{\infty} \in$ $L^{p^{\prime}}(S)$ and $\left\{h_{k}\right\}_{k=1}^{\infty} \in L^{p}(S)$ such that

$$
\sum_{k=-\infty}^{\infty}\left\|g_{k}\right\|_{L^{p^{\prime}}(S)}\left\|h_{k}\right\|_{L^{p}(S)}<\infty
$$

and for every $m \in \mathbb{Z}$

$$
\sigma(w, m)=(m(S))^{\frac{1}{2}}\left(e_{m}(w)\right)^{-1} \sum_{k=-\infty}^{\infty} h_{k}(w) \overline{\left(\mathcal{F}_{S} \overline{\bar{g}}\right)(m)}, w \in\left\{\mathfrak{s} \in S: e_{m}(\mathfrak{s}) \neq 0\right\} .
$$


Therefore,

$$
\begin{aligned}
& \left(T_{\eta} T_{\sigma} f\right)(s) \\
& =m(S) \sum_{n=-\infty}^{\infty} \eta(s, n) e_{n}(s) \int_{S} \overline{e_{n}(w)}\left(\sum_{m=-\infty}^{\infty} \sum_{k=-\infty}^{\infty} h_{k}(w) \overline{\left(\mathcal{F}_{S} \overline{g_{k}}\right)(m)}\left(\mathcal{F}_{S} f\right)(m)\right) d m(w) \\
& =m(S) \sum_{m=-\infty}^{\infty}\left[\sum_{k=-\infty}^{\infty} \sum_{n=-\infty}^{\infty} \eta(s, n) e_{n}(s)\left(\int_{S} h_{k}(w) \overline{e_{n}(w)} d m(w)\right) \overline{\left(\mathcal{F}_{S} \overline{g_{k}}\right)(m)}\right]\left(\mathcal{F}_{S} f\right)(m) \\
& =(m(S))^{3 / 2} \sum_{m=-\infty}^{\infty}\left[\sum_{k=-\infty}^{\infty} \sum_{n=-\infty}^{\infty} \eta(s, n) e_{n}(s)\left(\mathcal{F}_{S} h_{k}\right)(n) \overline{\left(\mathcal{F}_{S} \overline{g_{k}}\right)(m)}\right]\left(\mathcal{F}_{S} f\right)(m) \\
& =(m(S))^{1 / 2} \sum_{m=-\infty}^{\infty} e_{m}(s) \lambda(s, m)\left(\mathcal{F}_{S} f\right)(m),
\end{aligned}
$$

where

$$
\lambda(s, m)=(m(S))^{1 / 2}\left(e_{m}(s)\right)^{-1} \sum_{k=-\infty}^{\infty} h_{k}^{\prime}(s) \overline{\left(\mathcal{F}_{S} \overline{g_{k}}\right)(m)}
$$

and

$$
h_{k}^{\prime}(s)=(m(S))^{1 / 2} \sum_{n=-\infty}^{\infty} \eta(s, n) e_{n}(s)\left(\mathcal{F}_{S} h_{k}\right)(n)
$$

\section{Examples of $S$-operators AND $\mathbb{Z}$-OPERATORS}

In this section, we give necessary and sufficient conditions on symbols such that the corresponding $S$ operators and $\mathbb{Z}$-operators related to the particular finite measure spaces (defined in introduction section (1), 22 and (3)) is nuclear and formula for nuclear trace of $\mathbb{Z}$-operator.

Example 5.1. Consider the sequence of Legendre polynomials $\left\{P_{n}(x)=\frac{1}{2^{n} n !} \frac{d^{n}}{d x^{n}}\left(x^{2}-1\right)^{n}\right\}$ on $(-1,1)$. Then the set

$$
\left\{e_{n}=\frac{P_{n}}{\left\|P_{n}\right\|}=\sqrt{\frac{2 n+1}{2}} P_{n}\right\}_{n \geq 0}
$$

is an orthonormal basis for $L^{2}(-1,1)$. Thus the $S$-operator and $\mathbb{Z}$-operator corresponding to the above space can be written as follows:

$$
\left(T_{\sigma} f\right)(s)=\sqrt{2} \sum_{n \in Z} \sigma(s, n)\left(\mathcal{F}_{S} f\right)(n) \frac{P_{n}(s)}{\left\|P_{n}\right\|}
$$

where $\sigma:(-1,1) \times \mathbb{Z} \rightarrow \mathbb{C}$ is a measurable function and $f \in L^{2}(-1,1)$ and

$$
\left(T_{\tau} a\right)(n)=\frac{1}{\sqrt{2}} \int_{-1}^{1} \sigma(n, s)\left(\mathcal{F}_{\mathbb{Z}} a\right)(s) \frac{P_{n}(s)}{\left\|P_{n}\right\|} d s,
$$

where $\tau: \mathbb{Z} \times(-1,1) \rightarrow \mathbb{C}$ is a measurable function and $a \in L^{2}(\mathbb{Z})$. 
From Theorem 3.2 the $\mathbb{Z}$-operator $T_{\tau}: L^{p_{1}}(\mathbb{Z}) \rightarrow L^{p_{2}}(\mathbb{Z})$ is nuclear for $1 \leq p_{1}, p_{2}<\infty$ if and only if there exist sequences $\left\{g_{k}\right\}_{k=1}^{\infty} \in L^{p_{1}^{\prime}}(\mathbb{Z})$ and $\left\{h_{k}\right\}_{k=1}^{\infty} \in L^{p_{2}}(\mathbb{Z})$ such that

$$
\sum_{k=-\infty}^{\infty}\left\|g_{k}\right\|_{L^{p_{1}^{\prime}(\mathbb{Z})}}\left\|h_{k}\right\|_{L^{p_{2}(\mathbb{Z})}}<\infty
$$

and for all $n \in \mathbb{Z}$

$$
\tau(n, s)=\frac{1}{\sqrt{2 n+1}}\left(\overline{P_{n}(s)}\right)^{-1} \sum_{k=-\infty}^{\infty} h_{k}(n) \overline{\mathcal{F}_{\mathbb{Z}} \overline{g_{k}}(s)}, s \in\left\{\mathfrak{s} \in(-1,1): P_{n}(s) \neq 0\right\} .
$$

and the nuclear trace of $\mathbb{Z}$-operator is given by

$$
\begin{aligned}
\operatorname{tr}\left(T_{\tau}\right) & =\sum_{n=-\infty}^{\infty} \int_{-1}^{1} \tau(n, s)\left|e_{n}(s)\right|^{2} d s \\
& =\sum_{n=-\infty}^{\infty} \frac{2 n+1}{2} \int_{-1}^{1} \tau(n, s)\left|P_{n}(s)\right|^{2} d s .
\end{aligned}
$$

On the other hand, from Theorem 4.1 the $S$-operator $T_{\sigma}: L^{p_{1}}(-1,1) \rightarrow L^{p_{2}}(-1,1)$ is nuclear for $1 \leq p_{1}, p_{2}<\infty$ if and only if there exist sequences $\left\{g_{k}\right\}_{k=1}^{\infty} \in L^{p_{1}^{\prime}}(-1,1)$ and $\left\{h_{k}\right\}_{k=1}^{\infty} \in L^{p_{2}}(-1,1)$ such that

$$
\sum_{k=-\infty}^{\infty}\left\|g_{k}\right\|_{L^{p_{1}^{\prime}(-1,1)}}\left\|h_{k}\right\|_{L^{p_{2}(-1,1)}}<\infty
$$

and for all $n \in \mathbb{Z}$

$$
\sigma(s, n)=\frac{2}{\sqrt{2 n+1}}\left(P_{n}(s)\right)^{-1} \sum_{k=-\infty}^{\infty} h_{k}(s) \overline{\left(\mathcal{F}_{S} \overline{g_{k}}\right)(n)}, s \in\left\{\mathfrak{s} \in(-1,1): P_{n}(s) \neq 0\right\} .
$$

Remark 5.2. The sequence of normalized Hermite functions and the sequence of orthogonal Laguerre polynomials forms an orthonormal basis for $L^{2}(\mathbb{R})$ and $L^{2}(0, \infty)$ respectively as defined in (2) and (3). Similar to the above example, we also can find necessary and sufficient conditions on symbols such that the corresponding $S$-operators and $\mathbb{Z}$-operators related to these measure spaces are nuclear.

\section{ACKNOWLEDGEMENT}

The authors would like to thank the anonymous referee for his/her valuable suggestions. Vishvesh Kumar is supported by FWO Odysseus 1 grant G.0H94.18N: Analysis and Partial Differential Equations. Shyam Swarup Mondal thanks the Council of Scientific and Industrial Research, India, for providing financial support. He also thanks his supervisor Jitendriya Swain for his support and encouragement.

\section{REFERENCES}

[1] M. S. Agranovich, Spectral properties of elliptic pseudo-differential operators on a closed curve, Funct. Anal. Appl. 13, 279-281 (1971).

[2] S. Akoi, On the boundedness and the nuclearity of pseudo-differential operators, Comm. Partial Diff. Equations 6(8), 279-281 (1981). 
[3] L. Botchway, G. Kibiti and M. Ruzhansky, Difference equations and pseudo-differential operators on $\mathbb{Z}^{n}$, J. Funct. Anal. 278(11), 108473, 41pp (2020).

[4] D. Cardona, Weak-type $(1,1)$ bounds for a class of operators with discrete kernel, Rev. Integr. temas Mat. 33(1), 51-60 (2015).

[5] D. Cardona, On the nuclear trace of Fourier Integral Operators, Rev. Integr. temas Mat. 37(2), 219-249 (2019).

[6] D. Cardona and V. Kumar, Multilinear analysis for discrete and periodic pseudo-differential operators in $L^{p}$ spaces, Rev. Integr. temas Mat. 36(2), 151-164 (2018).

[7] D. Cardona and V. Kumar, $L^{p}$-boundedness and $L^{p}$-nuclearity of multilinear pseudo-differential operators on $\mathbb{Z}^{n}$ and the torus $\mathbb{T}^{n}$, J. Fourier Anal. Appl. 25(6), 2973-3017 (2019).

[8] D. Cardona, C. Del Corral and V. Kumar, Dixmier traces for discrete pseudo-differential operators, J. Pseudo-Differ. Oper. Appl. 11, 647-656 (2020).

[9] V. Catană, Z-operators related to a finite measure space, J. Pseudo Differ. Oper. Appl. 9(2), 173-188 (2018).

[10] V. Catană, S-operators related to a finite measure space, Applicable Analysis 99(2), 326-339 (2020).

[11] A. Dasgupta and V. Kumar, Hilbert-Schmidt and Trace class pseudo-differential operators on the abstract Heisenberg group, J. Math. Anal. Appl. 486(2), 123936 (2020).

[12] A. Dasgupta and M. W. Wong, Pseudo-differential operators on the affine group, Pseudodifferential operators: groups, geometry and applications Trends Math., Birkhaüser/Springer, Cham, 1-14 (2017).

[13] J. Delgado, The trace of nuclear operators on $L^{p}(\mu)$ for $\sigma$-finite Borel measures on second countable spaces. Integr. Equ. Oper. Theory 68(1), 61-74 (2010).

[14] J. Delgado, $L^{p}$ bounds for pseudo-differential operators on the torus, Operator Theory: Advances and Applications, 231, 103-116 (2013).

[15] J. Delgado and M. Ruzhansky, $L^{p}$-nuclearity, traces, and Grothendieck-Lidskii formula on compact Lie groups, J. Math. Pures Appl. 102(1), 153-172 (2014).

[16] J. Delgado and M. Ruzhansky and N. Tokmagambetov, Schatten classes, nuclearity and nonharmonic analysis on compact manifolds with boundary, J. Math. Pures Appl. 107(6), 758-783 (2017).

[17] J. Delgado and M. Ruzhansky, Fourier multipliers, symbols and nuclearity on compact manifolds, J. Anal. Math. 135(2), 757-800 (2018).

[18] J. Delgado and M. W. Wong, $L^{p}$-nuclear pseudo-differential operators on $\mathbb{Z}$ and $\mathbb{S}^{1}$, Proc. Amer. Math. Soc. 141(11), 3935-3942 (2013). 
[19] M.B. Ghaemi, M. Jamalpourbirgani and M. W. Wong, Characterization of nuclear pseudodifferential operators on $\mathbb{S}^{1}$ with applications to adjoints and products, J. Pseudo-Differ. Oper. Appl. 8, 191-201 (2017).

[20] M. B. Ghaemi, M. Jamalpourbirgani and M. W. Wong, Characterizations, adjoints and products of nuclear pseudo-differential operators on compact and Hausdorff groups, Politehn. Univ. Bucharest Sci. Bull. Ser. A Appl. Math. Phys. 79(4), 207-220 (2017).

[21] A. Grothendieck, Produits Tensoriels Toplogiques et Espaces Nucléaires, Memoirs Amer. Math. Soc. 16, (1955).

[22] A. Grothendieck, La théorie de Fredholm, Bull. Soc. Math. France 84, 319-384 (1956).

[23] L. Hörmander, The Analysis of Linear Partial Differential Operators. III, Springer-Verlag, Berlin (1985).

[24] M. Jamalpourbirgani, Characterization of nuclear pseudo-differential operator on $\mathbb{Z}$ with some applications, Math. Model. Nat. Phenom. 13(4), 17pp (2018).

[25] J. J. Kohn and L. Nirenberg, An algebra of pseudo-differential operators, Comm. Pure Appl. Math. 18, 269-305 (1965).

[26] V. Kumar, Pseudo-differential operators on homogeneous spaces of compact and Hausdorff groups, Forum Math. 31(2), 275-282 (2019).

[27] V. Kumar and S. S. Mondal, Schatten Class and nuclear pseudo-differential operators on homogeneous spaces of compact groups, (2019). https://arxiv.org/abs/1911.10554

[28] V. Kumar and M. W. Wong, $C^{*}$-algebras, $H^{*}$-algebras and trace ideals of pseudo-differential operators on locally compact, Hausdorff and abelian groups, J. Pseudo-Differ. Oper. Appl. 10(2), 269-283 (2019).

[29] V. Kumar and M. W. Wong, Correction to: $C^{*}$-algebras, $H^{*}$-algebras and trace ideals of pseudodifferential operators on locally compact, Hausdorff and abelian groups, J. Pseudo-Differ. Oper. Appl. (2020). https://doi.org/10.1007/s11868-020-00338-y

[30] S. Molahajloo and M.W. Wong, Pseudo-differential Operators on $\mathbb{S}^{1}$, In: New developments in pseudo-differential operators, Eds. L. Rodino and M.W. Wong, 297-306 (2008).

[31] S. Molahajloo, Pseudo-differential operators on $\mathbb{Z}$ : Pseudo-differential operators: complex analysis and partial differential equations, Oper. Theory. Adv. Appl. 205, 213-221 (2010).

[32] S. Molahajloo, A characterization of compact pseudo-differential operators on $\mathbb{S}^{1}$, Oper. Theory Adv. Appl. Birkhü̈er/Springer Basel AG, Basel. 213, 25-29 (2011).

[33] S. Molahajloo and M. Pirhayati, Traces of pseudo-differential operators on compact and Hausdorff groups, J. Pseudo-Differ. Oper. Appl. 4(3), 361-369 (2013). 
[34] V. Rabinovich, Exponential estimates of solutions of pseudo-differential equations on the lattice $(h \mathbb{Z})^{n}$ applications to the lattice Schrödinger and Dirac operators, J. Pseudo-Differ. Oper. Appl. 1(2), 233-253 (2010).

[35] V. Rabinovich and S. Roch, Essential spectra and exponential estimates of eigenfunctions of lattice operators of quantum mechanics, J. Phys. A 42(38), 21 pp (2009).

[36] J. A. Rempala, On the proof of the boundedness and nuclearity of pseudo-differential operators in $\mathbb{R}^{n}$, Annales Polonici Mathematici 52, 59-65 (1990).

[37] M. Ruzhansky and V. Turunen, On the Fourier analysis of operators on the torus. Modern trends in pseudo-differential operators, Birkhaüser, Basel, 87-105 (2007).

[38] M. Ruzhansky and V. Turunen, On the toroidal quantization of periodic pseudo-differential operators, Numer. Funct. Anal. Optim. 30(9-10), 1098-1124 (2009).

[39] M. Ruzhansky and V. Turunen, Quantization of pseudo-differential operators on the torus, $J$. Fourier Anal. Appl. 16(6), 943-982 (2010).

[40] M. Ruzhansky and V. Turunen, Pseudo-differential Operators and Symmetries: Background Analysis and Advanced Topics, Birkhaüser-Verlag, Basel (2010).

[41] M. Ruzhansky and V. Turunen, Global quantization of pseudo-differential operators on compact Lie groups, $S U(2)$, 3-Sphere, and homogebenous spaces, Int. Math. Res. Not. IMRN (11), 24392496 (2013).

[42] V. Turunen and G. Vainikko, On symbol analysis of periodic pseudodifferential operators, $Z$. Anal. Anwendungen. 17(1), 9-22 (1998).

[43] M.W. Wong, An Introduction to Pseudo-Differential Operators, 3rd edn., World Scientific, Singapore (2014).

VISHVESH KUMAR

Department of Mathematics: Analysis, Logic and Discrete Mathematics

GHent University

Krijgslaan 281, Building S8, B 9000 Ghent, Belgium .

E-mail address: vishveshmishra@gmail.com

SHYAM SWARUP MONDAL

Department of MATHEMATiCs

IIT GUWAHATI

Guwahati, Assam, India.

E-mail address: mondalshyam055@gmail.com 